\title{
EXPERIMENTAL VERIFICATION OF TEACHING TOPIC "THE RAINBOW FORMATION" IN SUBJECT PHYSICS AT HIGH SCHOOLS IN CITY HRADEC KRALOVE
}

\author{
Michal Musilek, Jan Šlegr \\ Faculty of Science, University of Hradec Králové, Czech Republic
}

\begin{abstract}
The authors from Faculty of Science University of Hradec Králové prepared worksheets for teaching topic "The rainbow formation" in subject physics at high school through laboratory work, which combines classical experiment and computer model. Classical experiment can be done with tools commonly available in the school science lab. A computer model based on geometric and physical analysis of the problem is to create a table and graph through spreadsheet software. Carefully prepared worksheet was provided to several teachers at different types of high schools in Hradec Kralove and the surrounding area. Addressed teachers used the methodology and worksheets for certain groups of students. Efficiency of the method was tested by pre-test and post-test and evaluated with method of normalized gain. Each of the teachers gave both tests to experimental and control group. The results confirm the hypothesis that high school students who have completed experimental teaching of the topic of "The rainbow formation" understand optical phenomena better than students who did not pass this teaching.
\end{abstract}

Key words: physics, optics, refraction of light, geometry, rainbow formation, computer, spreadsheet.

\section{Introduction}

Last year, the Department of Physics Faculty of Science University of Hradec Králové developed procedure enhancing the traditional high schools physics curriculum by the topic "The rainbow formation" (Halliday - Resnick - Walker, 1997) using information and communication technology. This process was broken down into a worksheet that connects classical experiment with a simple numerical model of the reflection of sunlight, i.e. a mix of visible monochromatic light with different wavelengths on tiny spherical drops. This model uses geometric and trigonometric functions and Snell's law for deriving the rainbow function and its modeling using a spreadsheet. Creating of the computer model of rainbow function was described in detail in the article How to include rainbow formation to teaching of physics at high schools using ICT? (Musílek, 2011).

Introductory classical experiment can be done as entry motivation with tools commonly available in the school science lab. We need spherical glass flask, water, rack and white drawing paper which serves as a focusing screen. Little limitation is the need of natural sunlight that reaches into the classroom through an open window. Sunlight goes into the glass bulb filled with water, which represents model of raindrop. Sunlight refracts 
on the flask surface, then is reflected from the inner side of the flask, and once again during exit out of the flask, exactly as sunbeams on the raindrops during creation of the natural rainbow. Protruding rays reaches classroom wall, which is in the shade. Because for

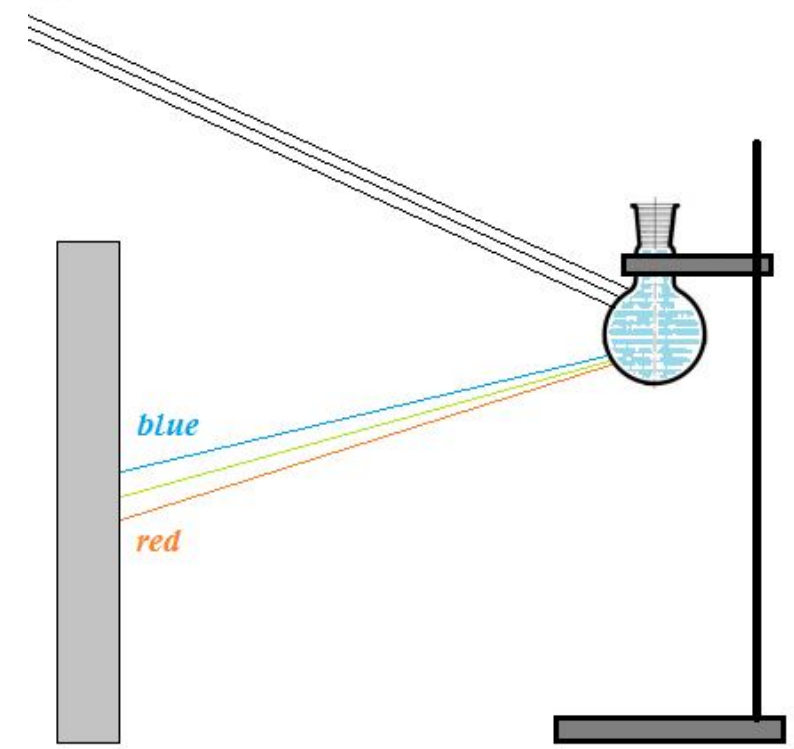

Figure 1: Introductory experiment. different wavelengths of light water has a different refractive index, draws incident rays on the focusing screen - drawing board, attached to the wall with adhesive tape - the solar spectrum decomposed into color bands that correspond individual monochromatic components of the light itself. See figure 1 .

Second part of the laboratory work is to derive the formula for the rainbow, or rather two relationships - for the primary and secondary rainbow function, based on the well-known geometric theorems and Snell's law, describing the refraction at the interface of two media. The following is the derivation for the primary rainbow. Derivation of the secondary rainbow is not any more complicated, so it isn't necessary also indicate it.

For the reflection is valid that angle of reflection is equal to the angle of impact i.e. $\alpha=\alpha^{\prime}$. For refraction is valid that the proportion of the sine of angle of impact to the sine of angle of refraction is equal to the relative index of refraction, i.e.

$$
\frac{\sin \alpha}{\sin \beta}=n
$$

This principle is often called Snell's law by its founder, Dutch mathematician and physician Willebrord Snell van Royen. It is also necessary to know the sine function and the inverse function (arcsine). And furthermore to know that the sum of the inner angles of triangle is always $180^{\circ}$ and that the correspondent top angles are equal. 


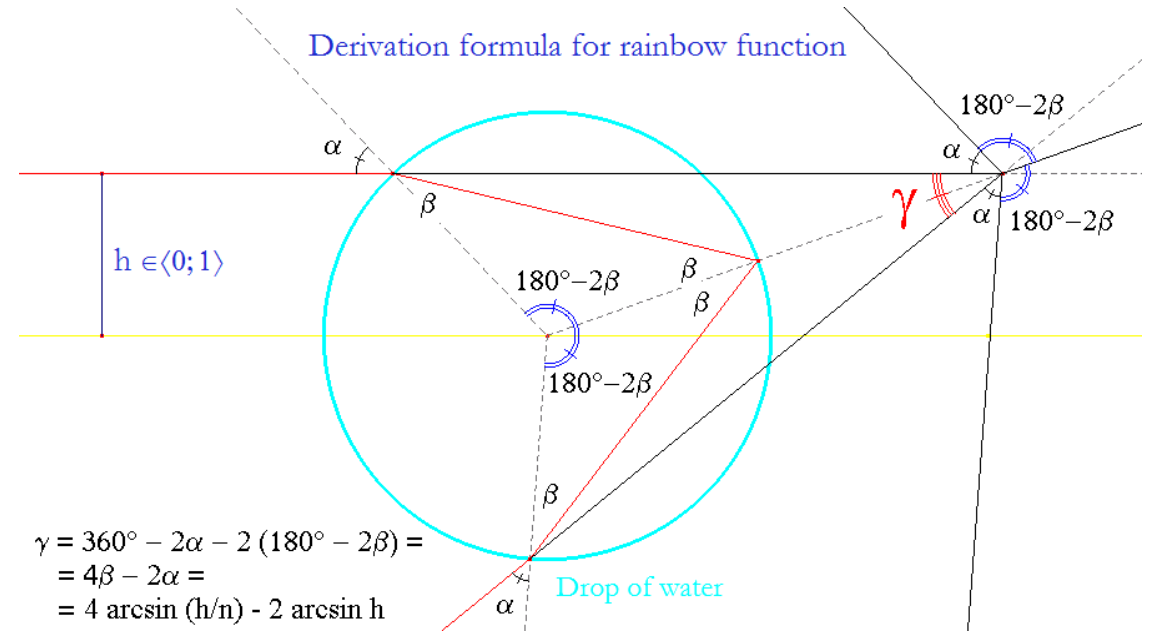

Figure 2: Derivation formula for rainbow function.

With the use figure 2 it is relatively easy to derive that for the resulting angle of refraction $\gamma$ on a drop of water is valid this relation:

$$
\gamma=4 \arcsin \left(\frac{h}{n}\right)-2 \arcsin (h),
$$

where $h$ is a relative distance of the dropping ray from the parallel ray crossing the centre of the drop (the real number from 0 to 1). The $n$ is the relative index of refraction for the transition of the ray from air to water. Because the absolute index of air refraction is normally very close to 1 (1.00026), it is possible to substitute the absolute index of the water refraction for $n$.

The third part of the laboratory work is the processing table and graph in a spreadsheet software, i.e. creating of own computer model of the rainbow. Derived rainbow function $\gamma$ is a real function of one real variable $h$, where index of refraction $n$ that depends on a wave length (and also on the colour) of the dropping light is a parameter. The process of function into three columns of the spreadsheet table for three different amounts of refraction index $n_{R}=1.330$ (red light), $n_{G}=1.334$ (green light), and $n_{B}=1.337$ (blue light). In the spreadsheet MS Excel 2010 we prepare amounts of the independent variable $h$ from 0 to 1 with the step of 0.01 . We can use the formula $=\left(4^{*} \operatorname{ARCSIN}(h / n)\right.$ $2 *(\operatorname{ARCSIN}(h))^{*} 180 / \mathrm{PI}()$ to the cell in the left top corner of the table. The formula figures out the amount of the function $\gamma$ in radians and by multiplying it with $180 / \pi$ it transfers it to the angle degrees. Then we copy the formula to the right and down to the whole area of the outputting amounts.

We use graph $\mathrm{X}-\mathrm{Y}$ with the smoothed connectors and with the grid for more precise deduction of amounts. We re-colour the colours of individual lines so that they correspond to the three chosen colours of the Sun light spectrum. 


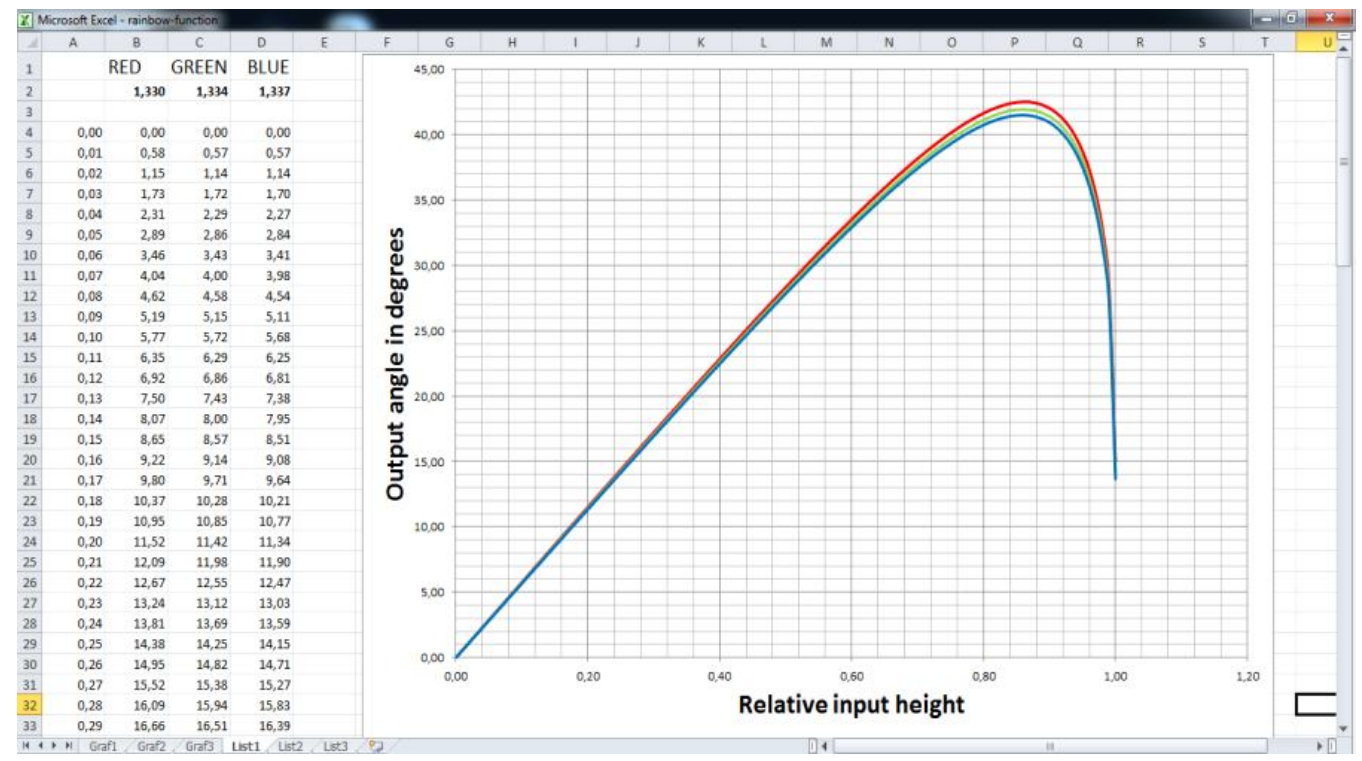

Figure 3: Calculation and display of rainbow function.

It is clear from the graph that the maximal angle of the light reflection on raindrops is $42.5^{\circ}$. It occurs in the red light and for the relative inputting height 0.86 . In the same inputting height we get $41.9^{\circ}$ for the green light and $41.5^{\circ}$ for the blue light. And this is the explanation of dispersion of the solar light to the colour spectrum and the formation of the beautiful atmospheric phenomenon that is called rainbow. The places that we can see in the parallel view with the solar rays under the certain angle crate an imaginary circle. Because a rainbow can be seen only when looking at a rainy screen in the sky, it has most often shape of a circle arc. We can see the arc with the Sun behind us and the rainy screen in front of us. If it rains only in the part of the sky in front of us, we can see only a part of the arc and the rainbow is touching the horizon only in one side or it doesn't touch it anywhere. Because all the light is reflecting on drops of water under the smaller angles, or under the limit angle, the inside area under the rainbow arc is lighter than the area outside the arc.

The experimental verification and evaluation of the proposed procedure, we asked several partner high schools in Hradec Králové and neighborhood.

\section{Methodology of Research}

Students were divided into two groups: The control group, where the topic of optics was taught in usual way, and the experimental group, where the above mentioned method was utilized. Both groups took the pre-test before the beginning of the teaching process and the post-test after the end of the topic of refractive index.

Pre-tests and post-test were evaluated with method of normalized gain, previously used by (Hake, 1998) as readily accessible, objective measure of learning in introductory physics. Normalized gain $G$ is defined as the change in score divided by the maximum possible increase: 


$$
G=\frac{\% \text { post-test }-\% \text { pre-test }}{100 \%-\% \text { pre-test }}
$$

Normalized gain $G \leq 0.3$ is commonly regarded as sign of not very effective learning, gain around 0.5 is considered average and $G \geq 0.7$ signify high efficiency of learning process.

Pre-test and post test were constructed in the same fashion and included for example this kind of questions:

The longest wavelength in the visible spectrum (around $700 \mathrm{~nm}$ ) belongs to
a) violet light,
b) blue light,
c) green light,
d) red light.

As the light passes through the medium with refractive index $n$,
a) the frequency of light decreases $n$-times,
b) the frequency of light increases $n$-times,
c) the velocity of light propagation in media decreases $n$-times,
d) the velocity of light propagation in media increases $n$-times.

Refractive index
a) depends only on the properties of the medium in which the light propagates,
b) depends only on the frequency of light,
c) depends on the properties of the medium and also on the frequency of light,
d) depends on the angle of incidence.

The last question is the most significant and demonstrates the understanding of the refractive index as function of wavelength.

In addition, teachers were asked to fill in the questionnaires, in which their opinions were collected and the activity of students during the activity rated.

\section{Results of Research}

The average normalized gain from all experimental groups at grammar schools was found to be 0.58 while for the control groups it was only 0.36 . For the other school types (for example medical school), the experimental group showed paradoxically lower normalized gain of only 0.14 while the control group had average gain of 0.36 . Our hypothesis that for some types of schools and some groups of students the traditional method is more applicable should be tested in subsequent research with wider variety of school types.

If we evaluate solely the question focused on the understanding of the wavelength dependence of refractive index (third question in the above excerpt), we can say that only $4 \%$ of students in experimental group answered it incorrectly in the post-test, while $36 \%$ of them answered it correctly in pre-test and also in the post-test. Remaining $60 \%$ of students answered it incorrectly in the pre-test and correctly in the post-test so we can say that this piece of knowledge was emphasized sufficiently with this activity. 
Evaluation of questionnaires filled in by teachers showed that teachers consider the topic of rainbow forming very important and the supplied material interesting and suitable for the support of the teaching process. At the grammar schools, the students (according to their teachers) worked during this activity in the same way as in any other laboratory exercise. At the medical school, students were more likely passive and they didn't like that they have to solve geometrical problems and work with spreadsheet editor.

\section{Discussion}

At all high schools involved in the project, except for just one, research has confirmed that the efficiency of the teaching process in the basics of optics will increase if the laboratory work "The rainbow formation" with ICT support was included. The inclusion of the topic "The rainbow formation" improves understanding of the physical phenomenon of refraction of light, including the dependence of the refractive index at the wavelength of light. The surprise was that on just one of the high schools the results were quite the opposite. Additionally, this school differed from the others with its focus. In this small sample, the results show a differential impact on the inclusion of the topic "The rainbow formation" non-traditional form of laboratory work for students of grammar schools then for students non-technical vocational high schools. Because the sample of students from vocational schools was too small, this argument can not be regarded as proven. We take it as a working hypothesis, which is necessary to verify or refute in further research.

The next stage will be completed by the existing research on the retention test and subsequently will be launched next stage of verification to more schools, and in particular the involvement of higher vocational schools with non-technical focus. Only then it will be possible to assess the effectiveness of inclusion on "The rainbow formation" in dependency on the type of secondary school.

\section{Conclusions}

The research results support the hypothesis that high school students who have completed the experimental teaching of the topic "The rainbow formation" understand optical phenomena better than students who did not pass this teaching. It is advisable to include laboratory work "The rainbow formation" with the support of ICT as a topic of teaching physics in secondary schools.

\section{Acknowledgements}

This paper is published thanks to the financial support of Specific project of Faculty of Science, University of Hradec Kralove. 


\section{References}

Halliday, David - Resnick, Robert Walker, Jearl. (2003). Fyzika. Vysokoškolská učebnice obecné fyziky. Translated from English original Fundamentals of Physics. Extended (5th edition, 1997). 1. vyd. VUTIUM a PROMTHEUS, Brno a Praha.

Musílek, M. (2011). Rainbow function [online]. c2011, last revision $25^{\text {th }}$ of September 2011 [cit. 2011-09-25] <http://www.musilek.eu/michal/rainbow/>.

Musilek, M. (2011). How to include rainbow formation to teaching of physics at high schools using ICT? Gamtamokslinis Ugdymas / Natural Science Education, 3 (32), 19-24.

Hake, Richard R. (1998). Interactive-engagement versus traditional methods: A six-thousandstudent survey of mechanics test data for introductory physics courses. American Journal of Physics, 66, 64-74.

Received 10 December 2012; accepted 26 February 2013

\section{Michal Musilek}

PhDr. Michal Musílek, Ph.D., vice-dean for education and internal relations, Faculty of Science, University of Hradec Králové, Rokitanského 62, 50003 Hradec Králové.

E-mail: michal.musilek@uhk.cz

Website: http://www.musilek.eu/michal/

\section{$\square$ Jan Šlegr}

RNDr., Doctoral Student at Department of Physics, Faculty of Science, University of Hradec Králové, Rokitanského 62, 50003 Hradec Králové.

E-mail: jan.slegr@uhk.cz

Website: http://lide.uhk.cz/pdf/student/slegrjal/ 